\title{
An experimental study of the dynamics of ascent of the bubble system in the presence of a surfactant
}

\author{
Alexandra Antonnikova ${ }^{1}$, Sergey Basalaev ${ }^{1, *}$, Anna Usanina $^{1}$, and Eugene Maslov ${ }^{1}$ \\ ${ }^{1}$ National Research Tomsk State University, 634050 Tomsk, Russia
}

\begin{abstract}
This paper presents investigations on the new experimental setup for obtaining a compact cluster of monodisperse bubbles of a given diameter is presented. Also we provided the results of experimental study of the bubble cluster floating-up in the presence of a surfactant in a wide range of Reynolds numbers. There was held a comparison of the dynamics of the floating-up of a monodisperse bubble cluster in a glycerol medium and in the medium glycerin supplemented with a surfactant.
\end{abstract}

\section{Introduction}

The behavior of fluid containing bubbles is distinctly different from behavior of homogeneous fluids under different physical and physical-chemical influence. These differences are abundantly used in industry - boiling, heat transfer in the two-phase mediums, cavitation, foaming, flotation. There is a question of the bubble cluster of given size generation in a number of problems, in particular, while researching an ignition of fluid electric discharge in fluids by way of specially created cavitational bubbles [1], while researching surfactants and acoustic waves on dynamics of bubble clusters [2, 3].

When two-phase flows with deformable particles of the disperse phase (liquid-drop, bubble flows) move, an important role is played by the properties of the interface, in particular, the surface tension coefficient. One of the physical factors affecting the boundary conditions is the presence of surfactants, which can change the criterial dependencies for the motion of the particle of the disperse phase by an order of magnitude. On the free boundary of a bubble or a droplet moving in a liquid medium with a surfactant, Marangoni tangential capillary forces arise, contributing to an increase in the coefficient of resistance of the particle. The problems arising in studying the patterns of motion and deformation of bubbles (or droplets) in a liquid containing a surfactant are formulated in the works $[4,5]$.

The regularities of the motion of bubbles in the presence of surfactants were investigated mainly for a single bubble [6, 7]. In contrast to the known papers, the results of an experimental study of the dynamics of the ascent of a set of spherical air bubbles in the presence of surface active substances in the Reynolds number range are presented $\mathrm{Re}=0.001 \div 30$. There was held a generalizing the data on the coefficient of resistance of a

\footnotetext{
* Corresponding author: tarm@niipmm.tsu.ru
} 
set of bubbles. Were used as a liquid: distilled water, water-glycerol solutions; as a surfactant: sodium dodecyl sulfate, potassium stearate.

\section{The scheme of the experimental setup}

An experimental setup for studying the dynamics of the emergence of a cluster of monodisperse bubbles in the presence of surfactants is shown in Fig. 1. The experimental setup comprises a reservoir 1 with a liquid located in the lower part, where air bubbles were produced by device 2 , connected by a tube 4 to a compressed air reservoir 5 , the pressure of which is supplied to the device 2 through a microreducer 6 with a control manometer 7 and a reducer 8 . In addition, there is a separate container 9 connected to the tube 4 through a reducer 10 and a tee 11 designed to create a positive pressure preventing the working fluid from flowing into the device 2 .

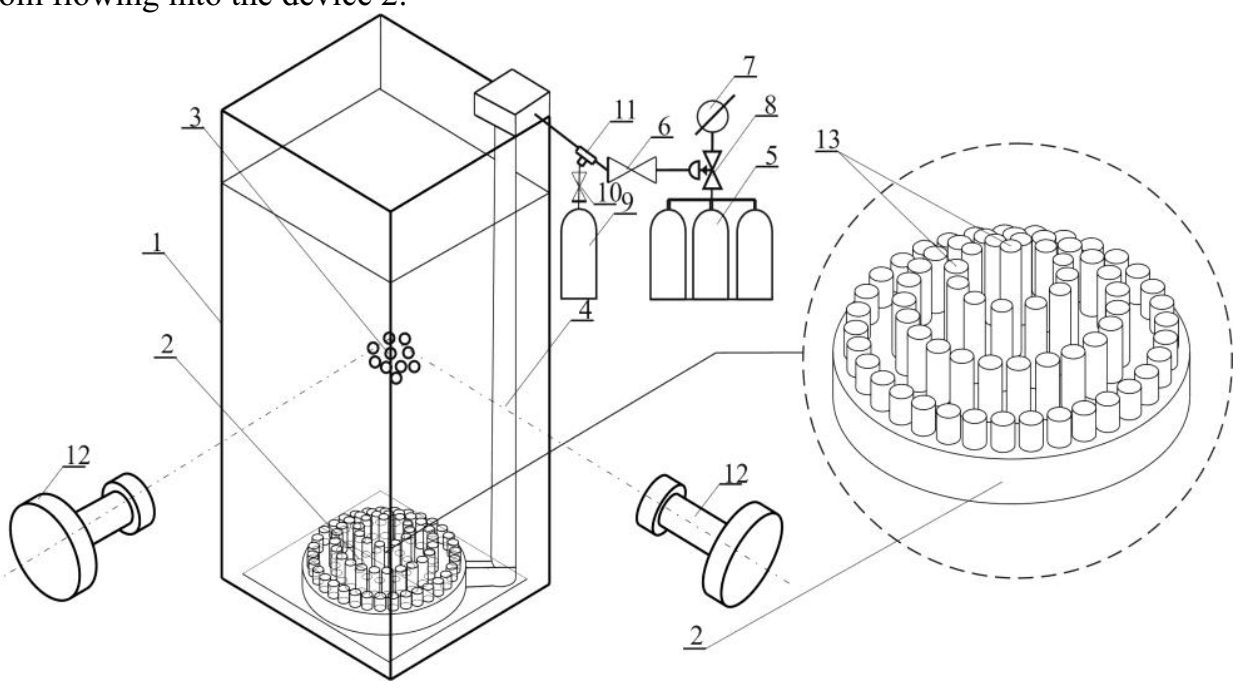

Fig. 1. The scheme of the experimental setup.

Receiver 1 is executed in the form of a cuvette with plane-parallel walls from optical glass of $0.3 \times 0.3 \times 0.6 \mathrm{~m}$ in size for visualization possibility of upfloat process of bubbles cluster. To visualize the dynamics of the bubble cluster 3 emergence in a liquid medium with the addition of surfactants, two high-speed video cameras 12 are used arranged to registration a bubble cluster in perpendicular planes.

The device for obtaining a monodisperse cluster of bubbles 2 is made in the form of a collector with perforations in the top cover starting from the center and along equidistant concentric circles, which makes it possible to obtain an axisymmetric bubble cluster. The use of microtubules of identical diameter 13, installed in perforations, provides the formation of monodisperse bubbles. Microtubes of the same height, located along each of the concentric circles, provide simultaneous formation of a "ring" of bubbles for each of the circles. The linear decrease in the height of the microtubes located on the circles, with an increase in the radius of the circle, ensures the sequential formation of each "ring" of bubbles with the same delay in time as they move away from the center of the collector cover, which makes it possible to obtain a compact cluster with a uniform spatial distribution of bubbles. 


\section{Results of the experimental study}

During the experimental studies, the number of bubbles $n=(1 \div 69)$ and their diameters $D=(3 \div 7) \mathrm{mm}$ in a monodisperse cluster varied, as well as the spatial arrangement of the bubbles among themselves [8]. Were used as a liquid: distilled water, water-glycerol solutions; as a surfactant: sodium dodecyl sulfate, potassium stearate.

The results of the visualization were given in a coordinate format for further analysis. In Fig. 2 shows a typical view of the spatial arrangement of bubbles at different times of ascent $(n=5, D=3.9 \mathrm{~mm})$.
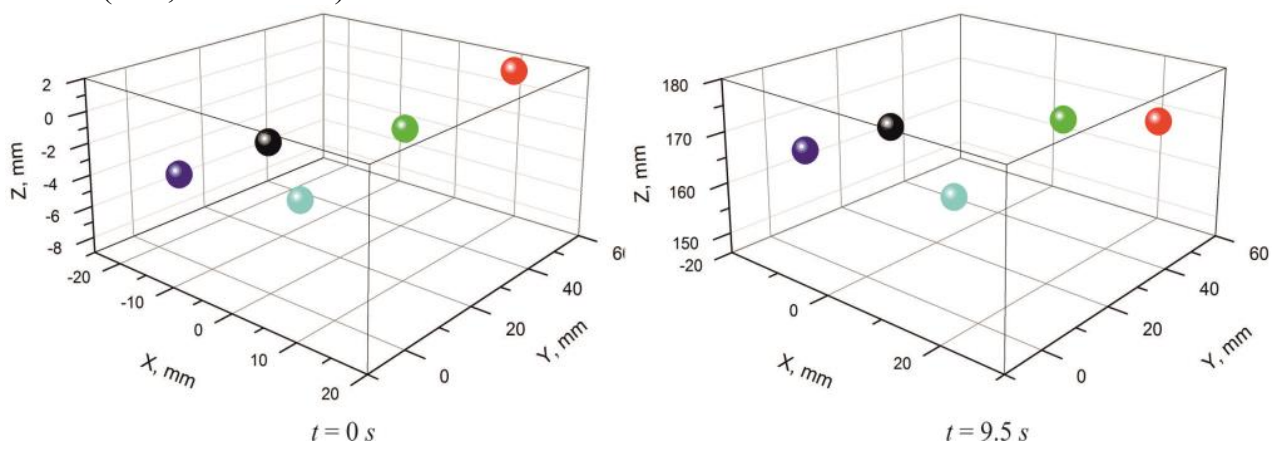

Fig. 2. Coordinates of the location of bubbles at different times $(t=0,9.5 \mathrm{~s})$.

The results of the experimental investigation for the emergence of a single bubble $(n=1)$ in the presence of surfactant are consistent with the results of known works on this topic and show that the bubble regime changes. The new bubble rise regime corresponds to the motion of a solid particle - the Stokes law at Reynolds numbers $\operatorname{Re}<1$ and the Klyachko law for $\operatorname{Re}>1$.

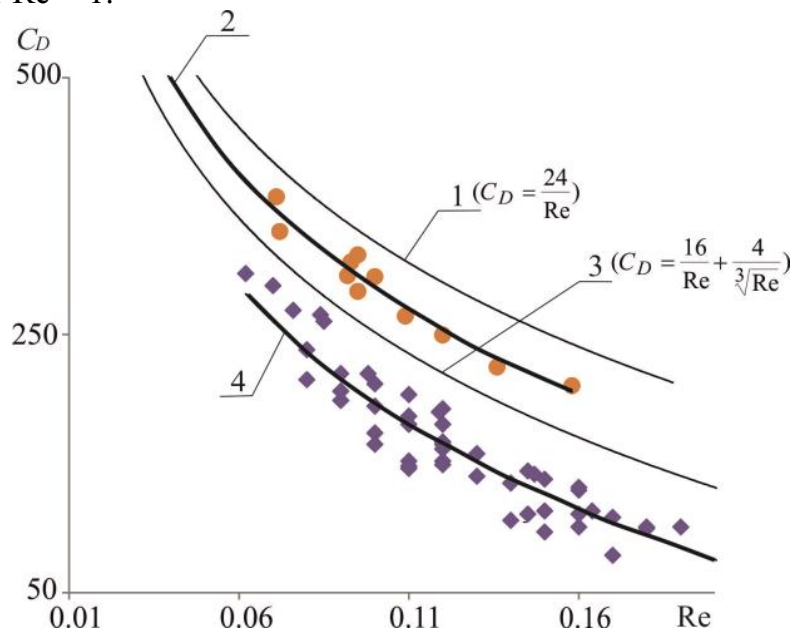

Fig. 3. Dependence of the resistance coefficient of a group of bubbles as a function of the Reynolds number ( 1 is the Stokes dependence; 2 is the empirical dependence, with the surfactant; 3 is the Hadamard-Rybczynski dependence; 4 is the empirical dependence, without surfactant).

To assess the effect of the presence of surfactant in a liquid on the dynamics of the ascent of a group of bubbles, two series of experiments were carried out: in a pure liquid (without surfactant) and in the presence of a surfactant (Fig. 3).

In experiments on the ascent of a group of bubbles in glycerin without surfactant additives, a value of a volume concentration $\mathrm{C}>0.002$ corresponding to the onset of the 
"partially blown" cloud. An empirical dependence is obtained for the coefficient of resistance of a group of bubbles $C_{D}$, moving in glycerine without surfactant additives in the $\operatorname{Re}<1$ region (curve 4 in Fig. 3):

$$
C_{D}=\frac{11.3}{\operatorname{Re}}
$$

The empirical dependence for the coefficient of resistance of a group of bubbles popping up in glycerin with the addition of surfactant has the form (curve 2 in Fig. 3):

$$
C_{D}=\frac{18.8}{\operatorname{Re}}
$$

It was found that the effect of surfactants on the dynamics of bubble emergence is determined by the Reynolds number. When $\operatorname{Re}<1$, the bubble surfacing regime change occurs at a lower concentration than at $\operatorname{Re}>1$.

\section{Conclusion}

- There is a certain critical value of the surfactant concentration in the liquid, at which the ascent bubble regime changes. In this case, the critical concentration value is a function of the bubble size (with increasing bubble size, the critical concentration value increases).

- The presence of surfactant leads to an increase in the coefficient of resistance by $33 \%$ and, accordingly, to a decrease in the ascent rate of a single bubble. In this case, the law of resistance changes from the Hadamard-Rybczynski formula to the Stokes formula.

- Empirical relationships for the coefficient of resistance of a group of bubbles ascent up in a liquid with a surfactant and without a surfactant are received.

- A decrease in the ascent rate of a group of bubbles in a liquid containing a surfactant has been found, compared with the velocity of a group of bubbles in a pure liquid.

This study was supported by the Russian Science Foundation (Project No. 15-19-10014).

\section{References}

1. A. P. Drozhzhin, S. M. Korobeynikov, B. C. Teslenko, Scientific bulletin of NGTU 2 (2003) [in Russian]

2. V. G. Levich, Physical and chemical hydrodynamics (Fizmatgiz, Moscow, 1959) [in Russian]

3. V. A Arkhipov, I. M. Vasenin, A. S. Usanina, Fl. Dyn. 51 (2), (2016)

4. R. Clift, J.R. Grace, M.E. Weber, Bubble. Drops and particles (Academic Press., London, 1978)

5. R. Griffith, Chem. Eng. Sc. 17 (1962).

6. J. Harper, App. Sci. Res. 38 (1982).

7. E. Almatroushi., A. Borhan, Ann. N.Y. Acad. Sci. 1027 (2004).

8. S. Goldaev, S. Basalaev, K. Afanasyev, MATEC Web Conf. 110, 01033 (2017) 